\title{
Lifshitz flows in IIB and dual field theories
}

\author{
Philipp Burda, ${ }^{1 a}$ Ruth Gregory ${ }^{a, b}$ and Simon F. Ross ${ }^{a}$ \\ ${ }^{a}$ Centre for Particle Theory, Durham University, \\ South Road, Durham, DH1 3LE, U.K. \\ ${ }^{b}$ Perimeter Institute, 31 Caroline Street North, \\ Waterloo, ON, N2L 2Y5, Canada \\ E-mail: philipp.burda@durham.ac.uk, r.a.w.gregory@durham.ac.uk, \\ s.f.ross@durham.ac.uk
}

ABSTRACT: We construct solutions describing flows between AdS and Lifshitz spacetimes in IIB supergravity. We find that flows from $\mathrm{AdS}_{5}$ can approach either $\mathrm{AdS}_{3}$ or Lifshitz in the IR depending on the values of the deformation from $\mathrm{AdS}_{5}$. Surprisingly, the choice between AdS and Lifshitz IR depends only on the value of the deformation, not on its character; the breaking of the Lorentz symmetry in the flows with Lifshitz IR is spontaneous. We find that the values of the deformation which lead to flows to Lifshitz make the UV field theory dual to the $\mathrm{AdS}_{5}$ geometry unstable, so that these flows do not offer an approach to defining the field theory dual to the Lifshitz spacetime.

KEYwords: Gauge-gravity correspondence, AdS-CFT Correspondence

ARXIV EPRINT: 1408.3271

\footnotetext{
${ }^{1}$ On leave of absence from ITEP, Moscow.
} 


\section{Contents}

1 Introduction $\quad 1$

2 Lifshitz and AdS solutions in five-dimensional gauged supergravity $\quad 3$

2.1 Ansatz for solutions and flows 4

$2.2 \mathrm{AdS}_{5}$ asymptotic solution $\quad 5$

$2.3 \mathrm{AdS}_{3} \times \mathcal{H}_{2}$ solution $\quad 6$

$2.4 \mathrm{Li}_{3} \times \mathcal{H}_{2}$ solution $\quad 6$

2.4.1 Tilded Lifshitz solution $z \geq 1 \quad 7$

$\begin{array}{lll}\text { 2.4.2 Untilded Lifshitz solution } 1 \leq z \leq 2 & 7\end{array}$

$\begin{array}{lll}3 & \text { RG } \text { flow solutions } & 7\end{array}$

3.1 Linearized analysis 8

$\begin{array}{llr}3.1 .1 & \text { Linearisation around } \mathrm{AdS}_{5} & 8\end{array}$

$\begin{array}{lll}3.1 .2 & \text { Linearisation around } \mathrm{AdS}_{3} \text { solutions } & 10\end{array}$

$\begin{array}{lll}3.1 .3 & \text { Linearisation around } \widetilde{\mathrm{Li}}_{3} \text { solutions } & 11\end{array}$

$\begin{array}{ll}\text { 3.1.4 Linearisation around } \mathrm{Li}_{3} \text { solutions } & 12\end{array}$

$\begin{array}{lll}3.2 & \text { Numerical Flows } & 12\end{array}$

3.2.1 Flows between $\mathrm{AdS}_{3}$ and $\mathrm{Li}_{3}$ spacetimes 12

$\begin{array}{lll}3.2 .2 & \text { Flows from } \mathrm{AdS}_{5} & 13\end{array}$

$\begin{array}{lll}3.3 & \text { Stability to condensation of supergravity fields } & 14\end{array}$

4 The UV field theory $\quad 16$

$\begin{array}{ll}\text { 4.1 UV field theory analysis } & 16\end{array}$

$\begin{array}{lll}4.2 & \text { Probe brane calculation } & 17\end{array}$

$\begin{array}{lll}4.3 & \text { Stability and Lifshitz dual field theories } & 19\end{array}$

$\begin{array}{ll}\text { A Additional AdS solutions } & 21\end{array}$

\section{Introduction}

The extension of holography [1] to field theories with dynamical exponent $z>1$ is interesting both for the potential application of these theories in condensed matter physics and for its potential to enlarge our understanding of holographic dualities (for reviews see e.g. $[2-4])$. Such theories have a symmetry under the scaling $t \rightarrow \lambda^{z} t, \vec{x} \rightarrow \lambda \vec{x}$, and it was realized in [5] that a holographic dual could be constructed by considering spacetimes with a metric

$$
d s^{2}=r^{2 z} d t^{2}-r^{2} d \vec{x}^{2}-\frac{d r^{2}}{r^{2}}
$$


which have an isometry under $t \rightarrow \lambda^{z} t, \vec{x} \rightarrow \lambda \vec{x}, r \rightarrow \lambda^{-1} r$. In [5, 6] simple "bottom-up" models admitting such solutions were proposed. They have since been realized as solutions in "top-down" models obtained from string theory: the case $z=2$ proves to be the simplest to realize [7-10], but a construction allowing for general values of $z$ was given in [11]. Some other particular values of $z$ were also realized in [12-14].

An interesting goal in such top-down constructions is to get a better understanding of the non-relativistic field theories dual to such Lifshitz solutions. It is particularly interesting to understand these holographic theories, as no examples of interacting theories with Lifshitz symmetries are known. In [15], holographic RG flows relating the Lifshitz and AdS solutions in the context of the massive IIA setup in [11] were constructed, and it was noted that the RG flows offered a potential approach to understanding the field theory dual to Lifshitz, as one could consider the flow from an AdS solution with a known dual to Lifshitz. Related work on such flows and their applications includes [16-24]. A dynamical interpolation was studied in [25]. A different approach to relating AdS to Lifshitz is [26, 27].

In this paper, we extend the work of [15] by considering flows involving the type IIB Lifshitz solutions in [11]. We start with the five-dimensional gauged supergravity obtained by compactifying IIB on an $S^{5}$, and consider further compactifying two spatial directions on a compact hyperbolic space, with certain gauge fluxes turned on on this space. There are asymptotically $\mathrm{AdS}_{5}$ solutions, where the proper size of compact hyperbolic space grows near the boundary, and $\mathrm{AdS}_{3}$ and 3-dimensional Lifshitz(denoted $\mathrm{Li}_{3}$ ) solutions where it has constant size. As in [15], we consider flows relating all these solutions. We focus particularly on the flows from $\mathrm{AdS}_{5}$, and analyze these in detail, identifying the deformation of $\mathrm{AdS}_{5}$ which source the flow and discussing its dual field theory description.

Working in the IIB context has two advantages: the field theory dual to the asymptotically $\mathrm{AdS}_{5}$ solution is the familiar $\mathcal{N}=4 \mathrm{SYM}$, and the deformation we are interested in includes as a special case a supersymmetric twist which has been previously studied in [28]. In the supersymmetric flow, [28] showed that the twist involves not only turning on a flux $Q$ but also adding a source $\lambda$ for a scalar operator transforming in the $\mathbf{2 0}$ of the $\mathrm{SU}(4) \mathrm{R}$-symmetry. We will see that the flows to non-supersymmetric $\mathrm{AdS}_{3}$ and Lifshitz geometries involve changing the values of $Q$ and $\lambda$ in a coordinated way: the flow reaches an IR fixed point on one-dimensional subspaces in the space of $\{Q, \lambda\}$ deformations.

Surprisingly, we do not need to turn on a source which breaks Lorentz symmetry explicitly in the UV to realize flows to Lifshitz: this Lorentz symmetry breaking will emerge spontaneously for appropriate values of $\{Q, \lambda\}$.

In [28], the deformation by $\{Q, \lambda\}$ was related to a change in the scalar Lagrangian in the $\mathcal{N}=4 \mathrm{SYM}$ theory, and it was shown to lead to flat directions for certain scalars in the supersymmetric case. We analyze this field theory Lagrangian deformation for our non-supersymmetric cases and find that there is a finite range of non-supersymmetric flows to $\mathrm{AdS}_{3}$ where the flat directions get lifted and the field theory scalars in the deformed field theory will be stable in the UV. Disappointingly, for the flows to $\mathrm{Li}_{3}$, the field theory deformation always leads to some runaway directions in the scalar space. These runaways correspond to brane nucleation instabilities in the bulk geometry (discussed for example in $[29,30])$, as we show explicitly by a probe brane calculation. Thus, for the flows to 
Lifshitz, the UV field theory is unstable, and this flow does not offer us a way to define the IR theory dual to the Lifshitz geometry. As in [15], we also find that for some values of $z$ the Lifshitz geometries have linearized modes which appear to violate the generalization of the Breitenlohner-Freedman bound [31]. These two types of instabilities do not appear to be related.

In section 2, we review the Romans 5D gauged SUGRA model [32] and review the Lifshitz solutions in this model [11], as well as discussing the families of $\mathrm{AdS}_{3}$ solutions. We then discuss the flows in section 3, first performing a linearized analysis about each of the solutions to determine the qualitative character of the flows and then numerically constructing the various flows. In section 4 , we analyze the deformation away from $\mathrm{AdS}_{5}$ in the UV and discuss the dual field theory.

\section{Lifshitz and AdS solutions in five-dimensional gauged supergravity}

We consider a consistent truncation of the $\mathcal{N}=4$ five-dimensional gauged supergravity theory obtained by reduction of the ten-dimensional type IIB supergravity on $S^{5}$, where we keep an $\mathrm{SU}(2) \times \mathrm{U}(1)$ subgroup of the $\mathrm{SU}(4)$ gauge group, and a single scalar $\phi$ [32]. This theory is a consistent truncation of the full higher dimensional theory, in the sense that any solutions in the 5D theory can be uplifted to Type IIB supergravity solutions in ten dimensions (see [33] for explicit detail).

The field content of the theory consists of the metric $g_{\mu \nu}, 5 \mathrm{D}$ dilaton field $\phi, \mathrm{SU}(2)$ gauge field $A_{\mu}^{(i)}, \mathrm{U}(1)$ gauge field $\mathcal{A}_{\mu}$ and two antisymmetric tensor fields $B_{\mu \nu}^{\alpha}$. The bosonic part of the Lagrangian is

$$
\begin{aligned}
\mathcal{L}= & -\frac{R}{4}+\frac{1}{2} \partial_{\mu} \phi \partial^{\mu} \phi-\frac{1}{4} \xi^{-4} \mathcal{F}_{\mu \nu} \mathcal{F}^{\mu \nu}-\frac{1}{4} \xi^{2}\left(F_{\mu \nu}^{(i)} F^{\mu \nu(i)}+B^{\mu \nu \alpha} B_{\mu \nu}^{\alpha}\right) \\
& +\frac{1}{4} \epsilon^{\mu \nu \rho \sigma \lambda}\left(\frac{1}{g_{1}} \epsilon_{\alpha \beta} B_{\mu \nu}^{\alpha} D_{\rho} B_{\sigma \lambda}^{\beta}-F_{\mu \nu}^{(i)} F_{\rho \sigma}^{(i)} \mathcal{A}_{\lambda}\right)+P(\phi),
\end{aligned}
$$

where $\xi=e^{\sqrt{\frac{2}{3}} \phi}$, the scalar field potential is

$$
P(\phi)=\frac{g_{2}}{8}\left(g_{2} \xi^{-2}+2 \sqrt{2} g_{1} \xi\right)
$$

and field strengths are

$$
\begin{aligned}
& \mathcal{F}_{\mu \nu}=\partial_{\mu} \mathcal{A}_{\nu}-\partial_{\nu} \mathcal{A}_{\mu}, \\
& F_{\mu \nu}^{(i)}=\partial_{\mu} A_{\nu}^{(i)}-\partial_{\nu} A_{\mu}^{(i)}+g_{2} \epsilon^{i j k} A_{\mu}^{(j)} A_{\nu}^{(k)} .
\end{aligned}
$$

The U(1) gauge coupling $g_{1}$ and $\mathrm{SU}(2)$ gauge coupling $g_{2}$ are two independent parameters of the theory. It was shown in [32] that these parameters can be eliminated by field redefinitions so that there are only three physically different theories, the $\mathcal{N}=4^{+}$theory, when $g_{1} g_{2}>0$, the $\mathcal{N}=4^{0}$ theory, when $g_{2}=0$, and the $\mathcal{N}=4^{-}$theory, when $g_{1} g_{2}<0$. We will consider here only the $\mathcal{N}=4^{+}$theory, i.e. we assume $g_{1} g_{2}>0$. We also set $B_{\mu \nu}^{\alpha}=0$ identically for all solutions and flows considered here. 
The equations of motion for the rest of the fields are then

$$
\begin{aligned}
R_{\mu \nu}= & 2 \partial_{\mu} \phi \partial_{\nu} \phi+\frac{4}{3} g_{\mu \nu} P(\phi)-\xi^{-4}\left(2 \mathcal{F}_{\mu \rho} \mathcal{F}_{\nu}^{\rho}-\frac{1}{3} g_{\mu \nu} \mathcal{F}_{\rho \sigma} \mathcal{F}^{\rho \sigma}\right) \\
& -\xi^{2}\left(2 F_{\mu \rho}^{(i)} F_{\nu}^{\rho(i)}-\frac{1}{3} g_{\mu \nu} F_{\rho \sigma}^{(i)} F^{\rho \sigma(i)}\right), \\
\square \phi= & \frac{\partial P}{\partial \phi}+\sqrt{\frac{2}{3}} \xi^{-4} \mathcal{F}_{\mu \nu} \mathcal{F}^{\mu \nu}-\sqrt{\frac{1}{6}} \xi^{2} F_{\rho \sigma}^{(i)} F^{(i) \rho \sigma}, \\
D_{\nu}\left(\xi^{-4} \mathcal{F}^{\nu \mu}\right)= & \frac{1}{4} \epsilon^{\mu \nu \rho \sigma \tau} F_{\nu \rho}^{(i)} F_{\sigma \tau}^{(i)}, \\
D_{\nu}\left(\xi^{2} F^{\nu \mu(i)}\right)= & \frac{1}{2} \epsilon^{\mu \nu \rho \sigma \tau} F_{\nu \rho}^{(i)} \mathcal{F}_{\sigma \tau} .
\end{aligned}
$$

\subsection{Ansatz for solutions and flows}

To construct flows, we only need to consider radial dependence of the bulk fields; we assume the holographic RG flow geometries we consider will preserve the translational invariance in the $t$ and $x$ directions, and will have the topological flux through the compact hyperbolic space. The most general ansatz we will need to consider is thus

$$
d s^{2}=e^{2 F(r)} d t^{2}-r^{2} d x^{2}-e^{2 d(r)} \frac{d r^{2}}{r^{2}}-e^{2 h(r)} \frac{d y_{1}^{2}+d y_{2}^{2}}{y_{2}^{2}}
$$

the $5 \mathrm{D}$ dilaton $\phi$ is also only a function of $r$, and we assume the gauge fields have at most nonzero $r-t$ or $r-x$ components. It is convenient to parametrize the fields in such a way as to eliminate geometric factors:

$$
\begin{aligned}
F_{r t}^{(3)} & =\frac{\tilde{A}(r)}{\xi r} e^{F+D}, & F_{r x}^{(3)} & =\frac{B(r)}{\xi} e^{D}, \\
\mathcal{F}_{r t} & =\frac{A(r) \xi^{2}}{r} e^{F+D}, & \mathcal{F}_{r x} & =\tilde{B}(r) \xi^{2} e^{D},
\end{aligned}
$$

where we have also introduced shifted and rescaled variables in order to eliminate $g_{1}$ and $g_{2}$ from all expressions:

$$
\begin{aligned}
& D(r)=d(r)+\frac{1}{3} \ln \left(g_{1} g_{2}^{2}\right) \\
& H(r)=h(r)+\frac{1}{3} \ln \left(g_{1} g_{2}^{2}\right) \\
& \varphi(r)=\xi^{3}(r) g_{1} g_{2}^{-1}
\end{aligned}
$$


Substituting all this into the equations (2.4) and introducing the new variable $\rho=\ln r$ we get

$$
\begin{aligned}
\frac{R_{t}^{t}}{g_{1}^{\frac{2}{3}} g_{2}^{\frac{4}{3}}} & =e^{-2 D}\left[F^{\prime}-F^{\prime} D^{\prime}+F^{\prime 2}+F^{\prime \prime}+2 H^{\prime} F^{\prime}\right] \\
& =\frac{1}{6}\left(\varphi^{-\frac{2}{3}}+2 \sqrt{2} \varphi^{\frac{1}{3}}\right)+\frac{4}{3}\left(A^{2}+\tilde{A}^{2}\right)+\frac{2}{3}\left(\tilde{B}^{2}+B^{2}\right)+\frac{2}{3} \varphi^{\frac{2}{3}} Q^{2} e^{-4 H} \\
\frac{R_{x}^{x}}{g_{1}^{\frac{2}{3}} g_{2}^{\frac{4}{3}}} & =e^{-2 D}\left[F^{\prime}-D^{\prime}+1+2 H^{\prime}\right] \\
& =\frac{1}{6}\left(\varphi^{-\frac{2}{3}}+2 \sqrt{2} \varphi^{\frac{1}{3}}\right)-\frac{2}{3}\left(A^{2}+\tilde{A}^{2}\right)-\frac{4}{3}\left(\tilde{B}^{2}+B^{2}\right)+\frac{2}{3} \varphi^{\frac{2}{3}} Q^{2} e^{-4 H} \\
\frac{R_{r}^{r}}{g_{1}^{\frac{2}{3}} g_{2}^{\frac{4}{3}}} & =e^{-2 D}\left[F^{\prime \prime}+F^{\prime 2}-F^{\prime} D^{\prime}-D^{\prime}+1-2 H^{\prime} D^{\prime}+2 H^{\prime 2}+2 H^{\prime \prime}\right] \\
& =\frac{-\varphi^{\prime 2}}{3 \varphi^{2} e^{2 D}}+\frac{1}{6}\left(\varphi^{-\frac{2}{3}}+2^{\frac{3}{2}} \varphi^{\frac{1}{3}}\right)+\frac{4}{3}\left(A^{2}+\tilde{A}^{2}-\tilde{B}^{2}-B^{2}\right)+\frac{2}{3} \varphi^{\frac{2}{3}} Q^{2} e^{-4 H} \\
\frac{R_{y_{1}}^{y_{1}}}{\frac{2}{3}} & =e^{-2 H}+e^{-2 D}\left[H^{\prime \prime}+2 H^{\prime 2}+H^{\prime} F^{\prime}+H^{\prime}-H^{\prime} D^{\prime}\right] \\
g_{1}^{3} & =\frac{1}{6}\left(\varphi^{-\frac{2}{3}}+2 \sqrt{2} \varphi^{\frac{1}{3}}\right)-\frac{2}{3}\left(A^{2}+\tilde{A}^{2}\right)+\frac{2}{3}\left(\tilde{B}^{2}+B^{2}\right)-\frac{4}{3} \varphi^{\frac{2}{3}} Q^{2} e^{-4 H}
\end{aligned}
$$

for the Einstein equations, where a prime now denotes $\partial_{\rho}$, and

$$
\begin{array}{rlrl}
\square \ln \varphi & =-e^{-2 D} \partial_{\rho}^{2} \ln \varphi-e^{-2 D} \partial_{\rho} \ln \varphi\left(1+F^{\prime}-D^{\prime}+2 H^{\prime}\right) \\
& =\frac{1}{2}\left(-\varphi^{-\frac{2}{3}}+\sqrt{2} \varphi^{\frac{1}{3}}\right)+4\left(\tilde{B}^{2}-A^{2}\right)-2\left(B^{2}-\tilde{A}^{2}\right)-2 \varphi^{\frac{2}{3}} Q^{2} e^{-4 H} \\
\partial_{\rho}\left(\varphi^{-\frac{2}{3}} r A e^{2 H}\right) & =2 \varphi^{-\frac{1}{3}} r B Q e^{D} ; & \partial_{\rho}\left(\varphi^{\frac{1}{3}} B e^{F+2 H}\right)=2 \varphi^{\frac{2}{3}} A Q e^{F+D} \\
\partial_{\rho}\left(\varphi^{\frac{1}{3}} r \widetilde{A} e^{2 H}\right) & =2 \varphi^{\frac{2}{3}} r \widetilde{B} Q e^{D} ; & \partial_{\rho}\left(\varphi^{-\frac{2}{3}} \widetilde{B} e^{F+2 H}\right) & =2 \varphi^{-\frac{1}{3}} \widetilde{A} Q e^{F+D} \\
A \widetilde{B}+\widetilde{A} B & =0
\end{array}
$$

for the 5D dilaton and gauge equations.

This system appears to involve eight unknown functions, but we see that in the Lifshitz solutions, one of the two sets of fluxes must be zero to satisfy (2.11), and therefore at most we turn on either the tilded or the untilded fluxes but never both. Thus, in a given flow we will have six unknown functions. These will be subject to seven equations: (2.8), (2.9), and two equations from (2.10). As usual, one of the equations in (2.8) is redundant because of the Bianchi identity.

\section{2 $\quad \mathrm{AdS}_{5}$ asymptotic solution}

In the ansatz (2.5), we have sliced our five dimensional space-time with two dimensional hyperbolic slices and $2+1$ dimensional planar slices. As such therefore, there is no solution for $F, D$, and $H$ which is globally $\mathrm{AdS}_{5}$, however, there are solutions which asymptote 
to $\mathrm{AdS}_{5}$ at large $r$, where the curvature of the hyperbolic space is effectively suppressed. These solutions will have

$$
F \sim \rho, \quad D \sim D_{0}, \quad H \sim H_{0}+\rho
$$

as $\rho \rightarrow \infty$, and will have a constant 5D dilaton, $\varphi \sim \varphi_{0}$, and vanishing gauge fluxes, $A \sim B \sim \widetilde{A} \sim \widetilde{B} \sim 0$ to leading order. Substituting this in (2.8), (2.9), (2.10), the leading order equations fix

$$
\begin{aligned}
4 e^{-2 D_{0}} & =\frac{1}{6}\left(\varphi_{0}^{-\frac{2}{3}}+2 \sqrt{2} \varphi_{0}^{\frac{1}{3}}\right), \\
0 & =\frac{1}{2}\left(-\varphi_{0}^{-\frac{2}{3}}+\sqrt{2} \varphi_{0}^{\frac{1}{3}}\right),
\end{aligned}
$$

which can easily be solved to find

$$
\varphi_{0}=\frac{1}{\sqrt{2}} \quad D_{0}=\frac{4}{3} \ln 2
$$

These asymptotically $\mathrm{AdS}_{5}$ solutions exist for any values of $H_{0}$ and the topological charge $Q$.

\section{$2.3 \quad \operatorname{AdS}_{3} \times \mathcal{H}_{2}$ solution}

In [28], a supersymmetric $\mathrm{AdS}_{3} \times \mathcal{H}^{2}$ solution was considered. Here we regard this as part of a one-parameter family of $\mathrm{AdS}_{3} \times \mathcal{H}^{2}$ solutions in the ansatz (2.5). In appendix A, we consider a more general two-parameter family of $\mathrm{AdS}_{3}$ solutions by turning on two fluxes.

We will get an $\mathrm{AdS}_{3} \times \mathcal{H}_{2}$ spacetime from the metric (2.5) by taking constant values for $H=H_{0}$ and $D_{0}$, and setting $F(\rho)=\rho$. It is easy to check that the system has such a solution for constant $5 \mathrm{D}$ dilaton field $\varphi_{0}$ and vanishing bulk gauge fluxes $A=\widetilde{A}=B=$ $\widetilde{B}=0$ if

$$
e^{-2 D_{0}}=\frac{\varphi_{0}^{\frac{1}{3}}}{2 \sqrt{2}}, \quad e^{-2 H_{0}}=\frac{1}{2 \varphi_{0}^{\frac{2}{3}}}, \quad Q^{2}=\varphi_{0} \sqrt{2}-1 .
$$

Therefore, we have a family of $\mathrm{AdS}_{3}$ solutions, parametrized by the value of $5 \mathrm{D}$ dilaton field $\varphi_{0}$, which should be in the range $\varphi_{0} \in\left[\frac{1}{\sqrt{2}}, \infty\right)$. These solutions are illustrated by a grey line in figure 1.

\section{$2.4 \quad \operatorname{Li}_{3} \times \mathcal{H}_{2}$ solution}

We now review the Lifshitz solutions obtained in [11]. As noted above, such solutions are obtained by taking either the tilded or untilded fluxes to vanish. The solutions are obtained from our ansatz by setting $F(\rho)=z \rho$, and taking constant functions $H=H_{0}$ and $D=D_{0}$ as in the $\mathrm{AdS}_{3}$ solutions. 


\subsubsection{Tilded Lifshitz solution $z \geq 1$}

If we turn on a tilded pair of gauge fluxes $\widetilde{A}=\widetilde{A}_{0}, \widetilde{B}=\widetilde{B}_{0}$ for some constant values $\widetilde{A}_{0}$ and $\widetilde{B}_{0},(A=B \equiv 0)$ then $(2.8),(2.9),(2.10)$ are satisfied if

$$
\begin{aligned}
\varphi_{0} & =\frac{\sqrt{2}(z+1)}{2 z^{2}+3 z-2}, & \widetilde{A}_{0}^{2} & =\frac{z(z-1)}{2} e^{-2 D_{0}}, \\
e^{-2 D_{0}} & =\left[2(z+1)^{2}\left(2 z^{2}+3 z-2\right)\right]^{-\frac{1}{3}}, & \widetilde{B}_{0}^{2} & =\frac{z-1}{2} e^{-2 D_{0}}, \\
e^{-2 H_{0}} & =\frac{3}{2} z e^{-2 D_{0}}, & Q^{2} & =\frac{2 z^{2}+3 z-2}{9 z} .
\end{aligned}
$$

This family of solutions is parametrized by the value of the dynamical exponent $z$, which in this case should be greater than one, and is shown in figure 1 as a blue line.

\subsubsection{Untilded Lifshitz solution $1 \leq z \leq 2$}

If we turn on the other pair of fluxes, i.e. untilded gauge fluxes $A=A_{0}, B=B_{0}$ for some constant values $A_{0}$ and $B_{0},(\widetilde{A}=\widetilde{B} \equiv 0)$ then $(2.8),(2.9),(2.10)$ are satisfied if

$$
\begin{aligned}
\varphi_{0} & =\frac{\sqrt{2} z(z+1)}{-2 z^{2}+3 z+2}, & A_{0}^{2} & =\frac{z(z-1)}{2} e^{-2 D_{0}}, \\
e^{-2 D_{0}} & =\left[2 z^{2}(z+1)^{2}\left(-2 z^{2}+3 z+2\right)\right]^{-\frac{1}{3}}, & B_{0}^{2} & =\frac{z-1}{2} e^{-2 D_{0}}, \\
e^{-2 H_{0}} & =\frac{3}{2} z e^{-2 D_{0}}, & Q^{2} & =\frac{-2 z^{2}+3 z+2}{9 z} .
\end{aligned}
$$

This second family of solutions is again parametrized by $z$, but this must now lie in the range $1 \leq z \leq 2$ which gives positive $Q^{2}$. These solutions are shown as a red line in the $\left(Q^{2}, \varphi_{0}\right)$ plane in figure 1.

\section{RG flow solutions}

We now turn to the construction of flows interpolating between the solutions reviewed in the previous section. Such interpolating solutions correspond to RG flows in the dual field theory, with the solution at small $r$ corresponding to the IR limit of the RG flow, and the solution at large $r$ corresponding the the UV limit of the RG flow. The study of such holographic flows was initiated in [34, 35].

Analogous flows were previously constructed for the Type IIA theory in [15]. As in that case, the charge $Q$ will be conserved along the flows; flows will move horizontally in figure 1. Therefore the solutions that can be related by flows are the $\widetilde{\mathrm{Li}}_{3}$ and $\mathrm{AdS}_{3}$ for large enough values of $Q$, and $\mathrm{AdS}_{3}$ and $\mathrm{Li}_{3}$ for smaller values of $Q$. There is also the possibility of having flows which start from the asymptotically $\mathrm{AdS}_{5}$ solution in the UV, which exists for any value of the charge $Q$, and approach any of these $\mathrm{AdS}_{3}$ or Lifshitz solutions in the IR. 


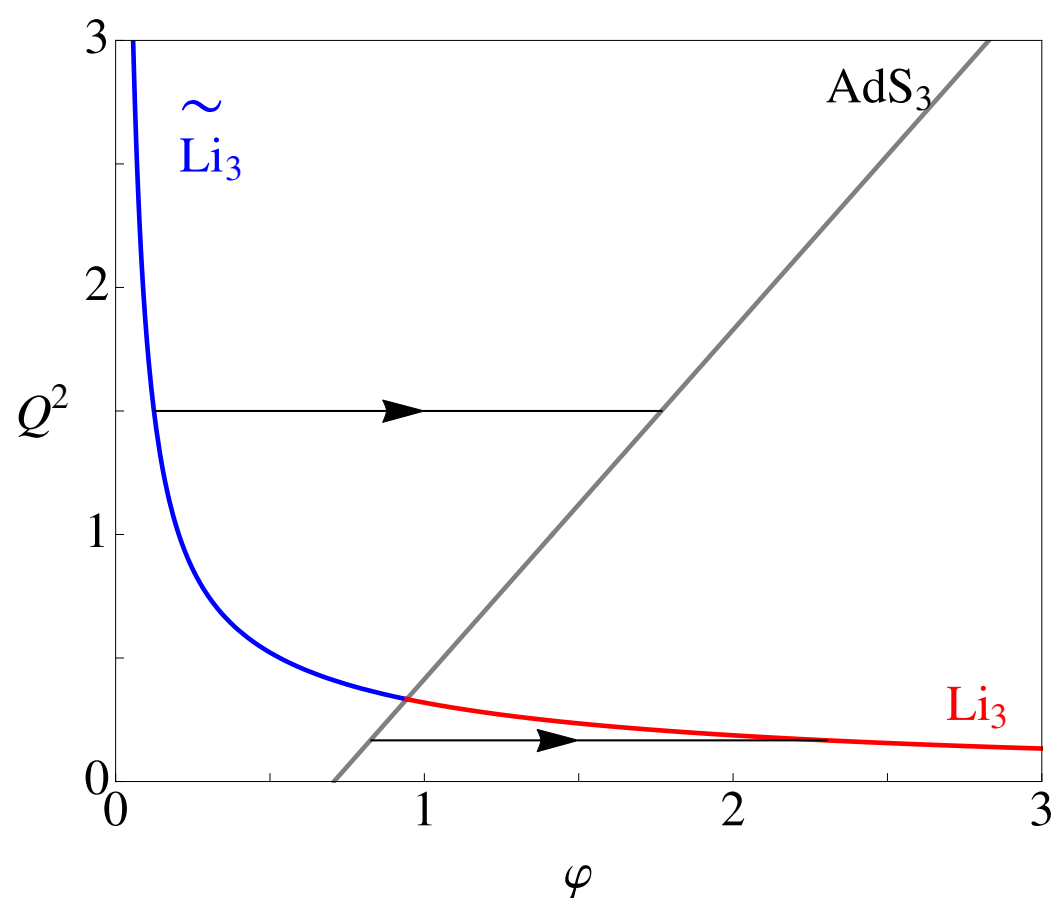

Figure 1. The values of $Q, \varphi_{0}$ for the $\mathrm{AdS}_{3}, \widetilde{L i_{3}}$ and $\mathrm{Li}_{3}$ solutions. The $\mathrm{AdS}_{3}$ family is parametrized by $\varphi_{0}$, which determines $Q^{2}=\sqrt{2} \varphi_{0}-1$. The Lifshitz families are parametrized by $z$, which determines $Q$ and $\varphi_{0}$. Also shown are flows between the solutions, which must occur at constant $Q$, with an arrow depicting the direction of the flow.

\subsection{Linearized analysis}

Before we proceed to the construction of the actual flows, we will perform a linearized perturbation analysis around each of the fixed-point solutions, to determine which direction we would expect the flows to go in (that is, which solution should be in the IR and which in the UV). This corresponds to computing the dimensions of the deforming operators in the dual field theories. We then construct the interpolating solutions numerically.

\subsubsection{Linearisation around $\mathrm{AdS}_{5}$}

The expansion around the asymptotically $\mathrm{AdS}_{5}$ solution is a little more conceptually involved than the others, because $\mathrm{AdS}_{5}$ is not an exact solution of the equations of motion, but only an asymptotic solution. We can avoid these subtleties by imagining that we take the radius of curvature of the compact hyperbolic space to zero by taking $h_{0} \rightarrow \infty$, and neglecting terms in the equations of motion involving $e^{-2 h_{0}}$. This will give us the linearized form of the equations of motion around the pure $\mathrm{AdS}_{5}$ solution which will allow us to read off the scaling of the linearized solutions. These scalings will remain valid for the linearized modes in the asymptotically $\mathrm{AdS}_{5}$ solution with finite $h_{0}$ to leading order at large $r$, as the physical volume of the compact hyperbolic space diverges as $r \rightarrow \infty$. 
We write the solution as

$$
\begin{aligned}
& \partial_{\rho} F=1+y_{0}(\rho), \quad D=D_{0}+y_{1}(\rho), \quad A=y_{8}(\rho), \\
& H=\rho+H_{0}+y_{2}(\rho), \quad \partial_{\rho} H=1+y_{4}(\rho), \quad B=y_{9}(\rho), \\
& \varphi=\varphi_{0}+y_{3}(\rho), \quad \partial_{\rho} \varphi=0+y_{5}(\rho),
\end{aligned}
$$

and linearize in the $y_{i}$, taking $H_{0} \rightarrow \infty$. At linear order we will not see the constraint (2.11), but we recall that we will only consider solutions with either $\left(y_{6}, y_{7}\right)$ or $\left(y_{8}, y_{9}\right)$, but not all four at the same time. The other equations in (2.8), (2.9), (2.10) then give us a system of first-order equations,

$$
\begin{array}{lll}
\dot{y}_{0}=-4 y_{0}, & \dot{y}_{1}=y_{0}-8 y_{1}+2 y_{4}, & \dot{y}_{2}=y_{4}, \\
\dot{y}_{3}=y_{5}, & \dot{y}_{4}=-4 y_{4}, & \dot{y}_{5}=-4 y_{3}-4 y_{5}, \\
\dot{y}_{6}=-3 y_{6}, & \dot{y}_{7}=-3 y_{7}, \quad \dot{y}_{8}=-3 y_{8}, & \dot{y}_{9}=-3 y_{9},
\end{array}
$$

and a constraint equation,

$$
y_{1}=\frac{y_{0}+2 y_{4}}{4} .
$$

We can easily verify that this constraint is consistent with the first-order system. Imposing the constraint, and keeping one of the two pairs of gauge fluxes, we will have a sevendimensional space of linearized solutions. For example, for the case where we keep $\left(y_{8}, y_{9}\right)$, the linearized solutions are

$$
\begin{aligned}
\partial_{\rho} F & =1+C_{0} e^{-4 \rho}, & \varphi & =\varphi_{0}+\lambda \rho e^{-2 \rho}+\eta e^{-2 \rho}, \\
D & =D_{0}+\frac{1}{4}\left(C_{0}+2 C_{4}\right) e^{-4 \rho}, & A & =C_{8} e^{-3 \rho}, \\
H & =\rho+H_{0}+C_{2}-\frac{1}{4} C_{4} e^{-4 \rho}, & B & =C_{9} e^{-3 \rho} .
\end{aligned}
$$

These solutions correspond to infinitesimal VEVs and sources for corresponding operators. The constants $C_{0}, C_{4}$ are the energy density and an anisotropic pressure; the corresponding sources are deformations of the boundary metric. These are $C_{2}$ and a constant $F_{0}$ in $F$, which we can freely add since the equations of motion only involve $\partial_{\rho} F$. Both $C_{2}$ and $F_{0}$ are pure gauge degrees of freedom; the former corresponds to shifting the background $H_{0}$, and the latter is a pure diffeomorphism. The parameters $C_{8}$ and $C_{9}$ are charge densities for the gauge fields; the corresponding sources are constant components of the vector potentials, which are pure gauge, and are also absent from our ansatz since we wrote it in terms of the field strengths. Finally $\lambda$ and $\eta$ are the source and VEV for the operator corresponding to the $5 \mathrm{D}$ dilaton. This operator is particularly interesting to us as we will see that the flows from $\mathrm{AdS}_{5}$ to the $\mathrm{AdS}_{3}$ and Lifshitz solutions will involve turning on this source. As this is a relevant deformation, we would expect flows from $\mathrm{AdS}_{5}$ in the UV, approaching the other solutions in the IR.

Since they do not enter into the equations of motion in our ansatz, the constant part of $F$ and the constant part of the gauge potentials will not play any role in the flows we consider. This is a remarkable fact; it implies that in the flows from $\mathrm{AdS}_{5}$ to Lifshitz, the only physical source we can find turned on at the $\mathrm{AdS}_{5}$ end of the flow is $\lambda$. This does not break the Lorentz invariance. Thus, when we have a flow to Lifshitz, the breaking of the Lorentz invariance along the flow is spontaneous. 


\subsubsection{Linearisation around $\mathrm{AdS}_{3}$ solutions}

We expect to have flows relating $\mathrm{AdS}_{3}$ to both $\widetilde{\mathrm{Li}_{3}}$ and $\mathrm{Li}_{3}$ spacetimes, therefore it is interesting to consider perturbations for both tilded and untilded fluxes in this case. Hence, we have the following linear perturbation from the $\mathrm{AdS}_{3}$ solution

$$
\mathbf{X}=\mathbf{X}_{0}+\mathbf{y}
$$

where $\mathbf{X}_{0}=\left(F^{\prime}, D, H, \varphi, H^{\prime}, \varphi^{\prime}, \widetilde{A}, \widetilde{B}, A, B\right)=\left(1, D_{0}, H_{0}, \varphi_{0}, 0,0,0,0,0,0\right)$ is the fixed point solution corresponding to the $\operatorname{AdS}_{3} \times \mathcal{H}_{2}$ spacetime and $\mathbf{y}(\rho)$ is a vector of perturbations. Linearising the equations of motion around the fixed point gives us a linear system

$$
\dot{\mathbf{y}}=\mathbb{A}_{A d S_{3}} \cdot \mathbf{y},
$$

together with a constraint equation analogous to (3.3). The matrix $\mathbb{A}_{A d S_{3}}$ is a $10 \times 10$ matrix dependent on the background field values, however, as with the $\mathrm{AdS}_{5}$ case, we may only switch on either the tilded or untilded fluxes, which both have exactly the same form of perturbation equations. In addition, the Bianchi identity implies a zero mode, thus our effective perturbations are reduced to a seven-dimensional system

$$
\dot{\mathbf{y}}_{\text {red }}=\mathbb{A}_{\text {red }} \cdot \mathbf{y}_{\text {red }}
$$

where $\mathbf{y}_{\text {red }}=\left(\delta F^{\prime}, \delta H, \delta \varphi, \delta H^{\prime}, \delta \varphi^{\prime}, \delta A(\delta \widetilde{A}), \delta B(\delta \widetilde{B})\right)$, and writing $c=\sqrt{2} / \varphi_{0}$ :

$$
\mathbb{A}_{\text {red }}=\left[\begin{array}{ccccccc}
-2 & 0 & 0 & 0 & 0 & 0 & 0 \\
0 & 0 & 0 & 1 & 0 & 0 & 0 \\
0 & 0 & 0 & 0 & 1 & 0 & 0 \\
0 & \frac{16-2 c}{3} & \frac{\sqrt{2} c}{9}(c-2) & -2 & 0 & 0 & 0 \\
0 & \frac{4 \sqrt{2}}{c}(c-2) & \frac{2-4 c}{3} & 0 & -2 & 0 & 0 \\
0 & 0 & 0 & 0 & 0 & -1 & \sqrt{4-2 c} \\
0 & 0 & 0 & 0 & 0 & \sqrt{4-2 c} & -1
\end{array}\right]
$$

In this format we see the perturbation of the flux decouples from the geometry, and the equation for $\delta F^{\prime}$ also decouples. This matrix has a set of eigenvalues $\left\{\Delta_{i}\right\}$,

$$
\Delta_{i}=-2 ; \quad-1 \pm \sqrt{4-c \pm \sqrt{9-2 c+c^{2}} ;} \quad-1 \pm \sqrt{4-2 c},
$$

with corresponding eigenvectors $\left\{\mathbf{v}_{i}\right\}$, thus the solution of the linear system (3.7) is

$$
\mathbf{y}_{\text {red }}=\sum_{i} \mathbf{v}_{i} e^{\Delta_{i} \rho} .
$$

The eigenvalues are plotted in figure 2, and we see that as in [15], some of the eigenvalues are complex for some values of $\varphi_{0}$, signalling a potential instability of these solutions. We will return to this issue at the end of our analysis.

Clearly, the $\Delta=-2$ eigenvalue corresponds to a pure geometry fluctuation, and actually corresponds to the fluctuation from a mass. The final pair of eigenvalues $\Delta_{ \pm}=$ 

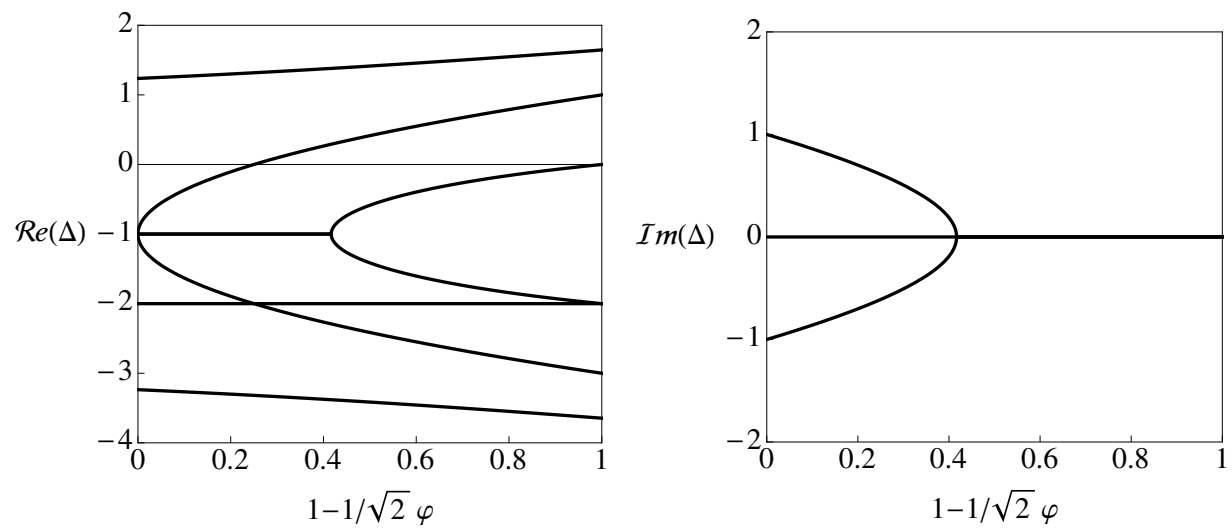

Figure 2. Plots of real and imaginary parts of the eigenvalues of the linear perturbations from the $\mathrm{AdS}_{3}$ solution as functions of the background value of the $5 \mathrm{D}$ dilaton field $\varphi_{0}$.

$-1 \pm \sqrt{4-\frac{2 \sqrt{2}}{\varphi_{0}}}$ switch on flux, hence corresponding operators on the field theory side are relevant when $\Delta_{+}<0$, i.e. for $\frac{1}{\sqrt{2}}<\varphi_{0}<\frac{2 \sqrt{2}}{3}$.

Note that $\varphi_{0}=\frac{2 \sqrt{2}}{3}$ corresponds exactly to the point where all $\mathrm{AdS}_{3}, \widetilde{\mathrm{Li}}_{3}$ and $\mathrm{Li}_{3}$ solutions coincide. Hence, for $\frac{1}{\sqrt{2}}<\varphi_{0}<\frac{2 \sqrt{2}}{3}$ we will have a relevant operator near $\mathrm{AdS}_{3}$. If we excite the untilded fluxes, we can then expect a flow from the $\mathrm{AdS}_{3}$ solution in the UV to the $\mathrm{Li}_{3}$ solution in the IR. For $\varphi_{0}>\frac{2 \sqrt{2}}{3}$ we will have an irrelevant operator near $\mathrm{AdS}_{3}$. So if we excite the tilded fluxes, we can expect to have flows from the $\widetilde{\mathrm{Li}}_{3}$ spacetime in the UV to the $\mathrm{AdS}_{3}$ spacetime in IR. These expected flows are presented in figure 1. We will construct these flows numerically below.

In addition to the flux deformations, we see from figure 2 that there is one deformation which is always irrelevant. This should correspond to the flow approaching $\mathrm{AdS}_{3}$ from the asymptotically $\mathrm{AdS}_{5}$ solution.

\subsubsection{Linearisation around $\widetilde{\mathbf{L i}}_{3}$ solutions}

In this case we must set the untilded fluxes to zero identically to satisfy (2.11). We write the variables as

$$
\mathbf{X}=\mathbf{X}_{0}+\mathbf{y}
$$

where $\mathbf{X}_{0}=\left(F^{\prime}, D, H, \varphi, H^{\prime}, \varphi^{\prime}, \widetilde{A}, \widetilde{B}\right)=\left(z, D_{0}, H_{0}, \varphi_{0}, 0,0, \widetilde{A}_{0}, \widetilde{B}_{0}\right)$ are the background values and $\mathbf{y}$ are the linear perturbations. This gives a linear system

$$
\dot{\mathbf{y}}=\mathbb{A}_{\widetilde{L i_{3}}} \cdot \mathbf{y}
$$

together with a constraint equation analogous to (3.3). The entries of the matrix $\mathbb{A}_{\widetilde{L i} i_{3}}$ are parametrized by the value of dynamical exponent $z$, and although the corresponding eigenvalues can be found analytically (in terms of square roots of solutions to a cubic) their form is not particularly illuminating thus we present them only graphically in figure 3 . The eigenvalues occur in pairs with the sum of each pair equal to $-(z+1)$. We see that we have complex eigenvalues for all values of $z$ along this family. We also note that there is a 


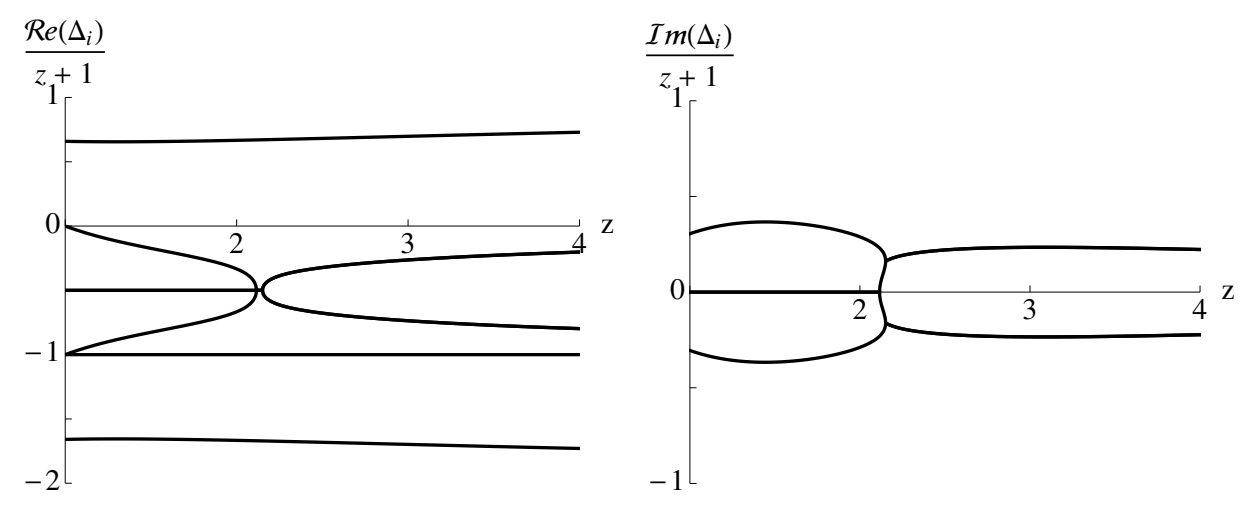

Figure 3. Plots of the real and imaginary parts of the eigenvalues of the linear perturbations from the $\widetilde{\mathrm{Li}}_{3}$ solutions, divided by $z+1$, as functions of the background values of the dynamical exponent $z$.

single irrelevant mode, corresponding to the expected flow approaching this solution from the asymptotically $\mathrm{AdS}_{5}$ solution.

\subsubsection{Linearisation around $\mathrm{Li}_{3}$ solutions}

This is similar to the previous case, although now it is the tilded fluxes which must be set equal to zero. We again have an 8-dimensional system of linear perturbations, with background values $\mathbf{X}_{0}=\left(F^{\prime}, D, H, \varphi, H^{\prime}, \varphi^{\prime}, A, B\right)=\left(z, D_{0}, H_{0}, \varphi_{0}, 0,0, A_{0}, B_{0}\right)$, and a linear system with a matrix $\mathbb{A}_{L i_{3}}$ and a constraint. We will again have seven linearly independent modes, with eigenvalues coming in pairs, with the sum of the eigenvalues in each pair equal to $-(z+1)$. The resulting eigenvalues are presented in figure 4 . Here we see complex eigenvalues for a range of values of $z$ near 1 , but there is a range near 2 where all the eigenvalues are real and the solutions may be stable. We also note that there are two irrelevant modes, corresponding to the expected flows approaching this solution from asymptotically $\mathrm{AdS}_{5}$ and $\mathrm{AdS}_{3}$ solutions.

\subsection{Numerical Flows}

Here we present the result of numerical solutions of the full non-linear system of equations of motion for the interpolating solutions between different fixed points in UV $(r \rightarrow \infty)$ and IR $(r \rightarrow 0)$. We discuss first the flows between $\mathrm{AdS}_{3}$ and $\mathrm{Li}_{3}$ spacetimes and then consider the flows from the asymptotically $\mathrm{AdS}_{5}$ solution in the UV.

\subsubsection{Flows between $\mathrm{AdS}_{3}$ and $\mathrm{Li}_{3}$ spacetimes}

From the linearized analysis, we expect flows from $\mathrm{AdS}_{3}$ in the UV to $\mathrm{Li}_{3}$ in the IR and flows from $\widetilde{\mathrm{Li}}_{3}$ in the UV to $\mathrm{AdS}_{3}$ in the IR, as depicted in figure 1. We constructed examples of these flows numerically, using a shooting method. The shooting is carried out starting from the IR fixed point at small $r$, integrating numerically to larger $r$. Shooting is required to obtain the flows between $\mathrm{AdS}_{3}$ and $\mathrm{Li}_{3}$ because the IR fixed point always has two positive eigenvalues, and the generic flow will go to the asymptotically $\mathrm{AdS}_{5}$ solution. Hence possible directions of shooting lie in the plane spanned by the two corresponding 

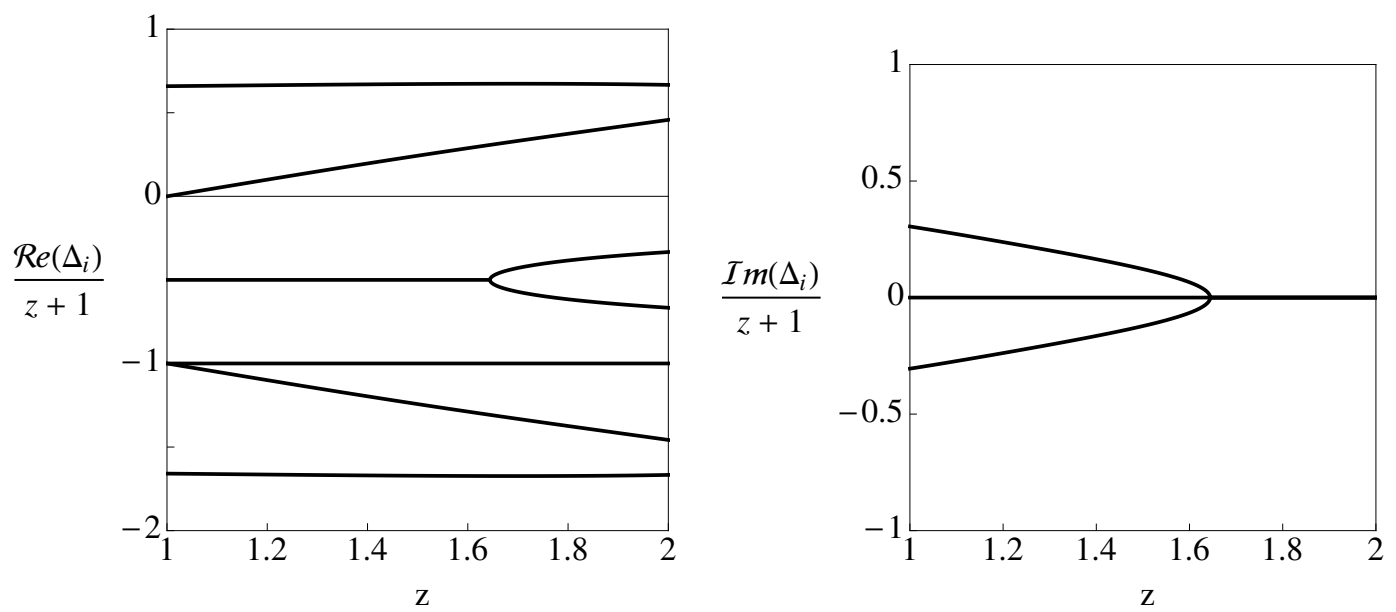

Figure 4. Plots of the real and imaginary parts of the eigenvalues of linear perturbations from the $\mathrm{Li}_{3}$ solutions, divided by $z+1$, as functions of the background values of the dynamical exponent $z$, in this case $1 \leq z \leq 2$.
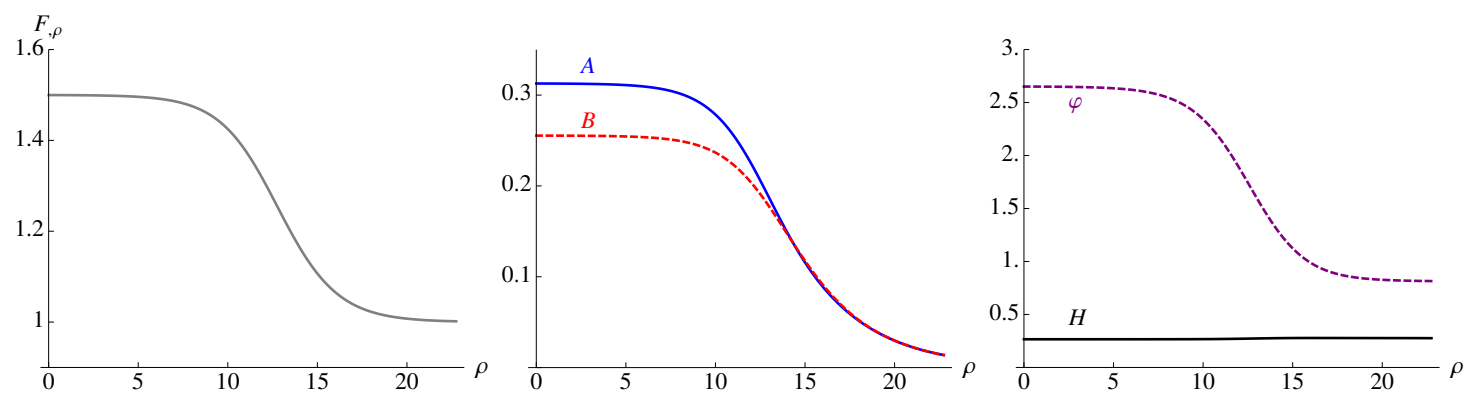

Figure 5. Solution interpolating between $\mathrm{Li}_{3}$ with $z=3 / 2$ and $\operatorname{AdS}_{3}$, with $Q^{2}=\frac{4}{27}$.

unstable directions and can be parametrized by the single angle variable, say, $\zeta$. We find the value of $\zeta$ giving the desired flow by bisection of an initial interval of values of $\zeta$.

- $Q^{2} \in\left[0, \frac{1}{3}\right]:$ Flows from $\mathrm{AdS}_{3}$ to $\mathrm{Li}_{3}$

We present an example of such a solution in figure 5: this case interpolates between the untilded Lifshitz solution with $z=3 / 2$ for small $r$ (IR) and the $\mathrm{AdS}_{3}$ solution for large $r$ (UV) . The plot of $F^{\prime}$ shows that it starts from the value $3 / 2$ and goes to 1 , the other plots show how fluxes of the gauge fields go to zero at large $r$.

- $Q^{2}>\frac{1}{3}$ : Flows from $\widetilde{\mathrm{Li}_{3}}$ to $A d S_{3}$

We present an example of such a solution in figure 6: this case interpolates between $\mathrm{AdS}_{3}$ for small $r$ (IR) and the $\widetilde{\mathrm{Li}}_{3}$ solution with $z=2$ for large $r(\mathrm{UV})$. The plot of $\partial_{\rho} F$ shows that it starts from 1 and goes to the value 2, the other plots show how fluxes of the gauge fields grow, approaching constant values at large $r$.

\subsubsection{Flows from AdS $_{5}$}

The flows which approach the asymptotically $\mathrm{AdS}_{5}$ solution in the UV and end at $\mathrm{AdS}_{3}$ or $\mathrm{Li}_{3}$ in IR are easy to construct numerically, integrating outward from the IR. We find 

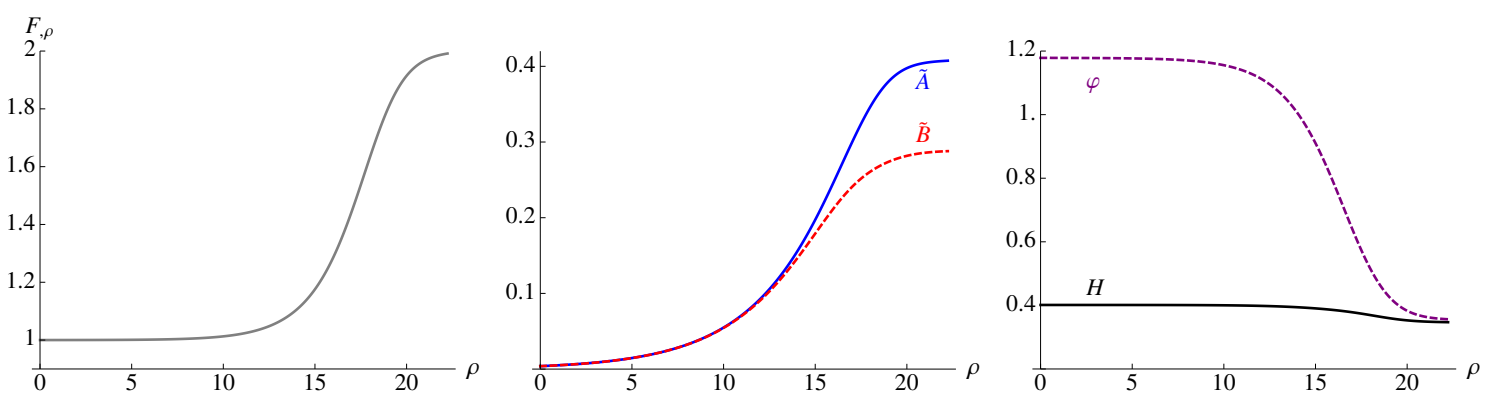

Figure 6. Solution interpolating between $\mathrm{AdS}_{3}$ and $\widetilde{\mathrm{Li}}_{3}$ with $z=2$, with $Q^{2}=\frac{2}{3}$.

that the endpoint of the flow from $\mathrm{AdS}_{5}$ is uniquely determined by the pair $\{Q, \lambda\}$, where $\lambda$ is the coefficient in front of the slow fall-off mode in the expansion of the $5 \mathrm{D}$ dilaton field near the $\mathrm{AdS}_{5}$ solution,

$$
\varphi=\frac{1}{\sqrt{2}}+\frac{\lambda}{r^{2}} \ln r+\frac{\eta}{r^{2}}+\ldots
$$

On the field theory side, $\lambda$ corresponds to the source of an operator $\mathcal{O}_{2}$, as discussed in Maldacena and Nunez [28], however, for future reference we note that the deformation parameter used there, $\bar{\lambda}$, is related to our $\lambda$ via

$$
\bar{\lambda}=\frac{\sqrt{2}}{3} e^{2 h_{0}} \lambda
$$

This operator (together with the curvature of the $\mathcal{H}_{2}$ and the flux $Q$ ) induces the RG flow on the field theory side. As noted previously, the fact that these flows only involve turning on a source for this operator implies that the flows to Lifshitz spacetimes break the Lorentz invariance spontaneously.

The values of $\bar{\lambda}$ for which we flow to the different solutions are presented schematically in figure 7. If we move along the $\mathrm{AdS}_{3}$ (grey) line in the direction of increasing of $Q$, then the corresponding value of $\bar{\lambda}$ is also increasing. For $Q=0 \bar{\lambda}=0$, while for $Q=1 \bar{\lambda}=\frac{1}{6}$; this latter value corresponds to the supersymmetric flow of [28]. If we move along the $\widetilde{\mathrm{Li}_{3}}$ (blue) line up (in the direction of increasing $Q$ and also increasing $z$ ), then the corresponding value of $\bar{\lambda}$ is decreasing, in such a way that for $Q=\sqrt{\frac{2}{3}}(z=2) \bar{\lambda}=0 .{ }^{1}$ Above this point $\bar{\lambda}<0$. If we move along the $\mathrm{Li}_{3}$ (red) line down (in the direction of decreasing $Q$, but increasing $z$ ), then the corresponding value of $\bar{\lambda}$ is increasing. Numerically, $\bar{\lambda} \rightarrow \frac{1}{6}$ as $z \rightarrow 2(Q \rightarrow 0)$. We will discuss the field theoretic implications of the values of $\bar{\lambda}$ in the next section, but first comment on stability of the supergravity solutions.

\subsection{Stability to condensation of supergravity fields}

In the analysis of the linearized perturbations, we encountered some complex eigenvalues for some values of parameters, as in the analysis of the IIA case in [15]. For a decoupled scalar, such complex eigenvalues appear when the scalar violates the BreitenlohnerFreedman bound, and there is then an instability to condensation of the scalar. We would

\footnotetext{
${ }^{1}$ This is a numerical result, but it seems very reasonable, because in Lifshitz theories, a theory with $z=2$ always was a special case.
} 


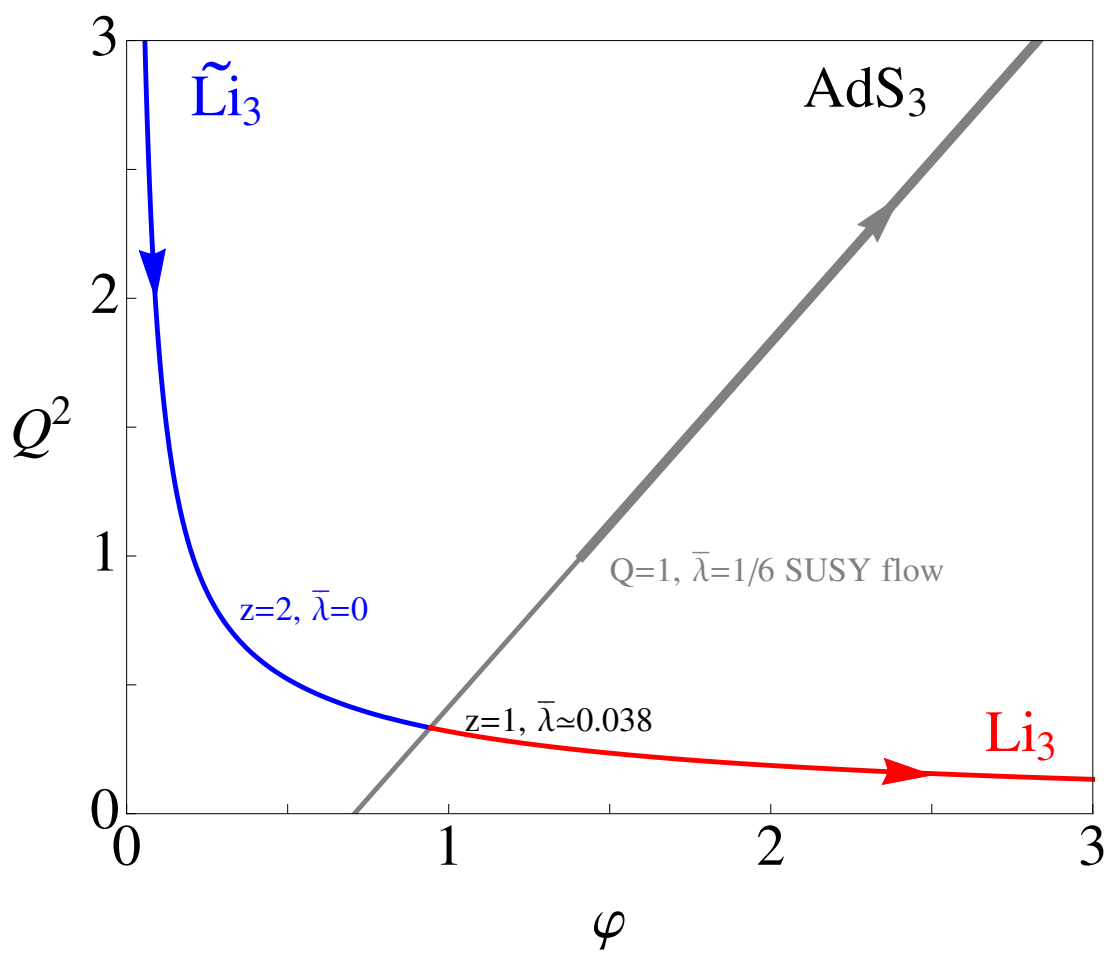

Figure 7. Plots of $\mathrm{AdS}_{3}, \widetilde{L i_{3}}$ and $\mathrm{Li}_{3}$ solutions, indicating the corresponding value of $\bar{\lambda}$ in the asymptotically $\mathrm{AdS}_{5} \mathrm{UV}$ region in the flow solutions. The arrows indicate the direction of increas$\operatorname{ing} \bar{\lambda}$.

expect that there will be a similar instability to condensation of the modes with complex eigenvalues in our case, although we will not attempt to carry out a time-dependent analysis to demonstrate this instability explicitly. Certainly the appearance of the complex eigenvalues obstructs the usual interpretation of the eigenvalue as the dimension of the corresponding operator in the field theory.

Also, it was noted in [36] that purely from a bulk spacetime perspective, when such complex eigenvalues appear for a scalar field there is no boundary condition which preserves the inner product which is invariant under the Lifshitz scaling isometry. Thus, we expect that in the cases with complex eigenvalues, we simply cannot choose boundary conditions such that our bulk solution is dual to an anisotropic scaling invariant field theory with a conserved inner product.

A nice field theory dual description of the fixed points with complex eigenvalues is thus unlikely to exist. This leaves as potentially interesting cases a range of the $\mathrm{AdS}_{3}$ fixed points and a range of the untilded $\mathrm{Li}_{3}$ fixed points with $z$ near 2. This is an interesting range of Lifshitz solutions, and an improvement of the IIA case, where the Lifshitz solutions with no complex eigenvalues were at larger values of $z$. 


\section{The UV field theory}

Our interest in studying flows, particularly those from asymptotically $\mathrm{AdS}_{5}$ spacetimes, is mainly that they might help us to understand the field theories dual to these spacetimes. In this section, we consider some stability issues that can obstruct our ability to learn about the field theory from these flows. For field theory on a flat space, the scalars in the adjoint of $\mathrm{SU}(\mathrm{N})$ have flat directions corresponding to the Coulomb branch. However in our class of spacetimes, we are compactifying two of the directions on which the field theory lives on a space of negative curvature. One might therefore expect the curvature coupling of the field theory scalars to produce a runaway instability for the diagonal components of these scalar matrices. From the bulk spacetime point of view, the diagonal components of the scalars are positions of branes, so this runaway would be a brane nucleation instability.

The story is of course more complicated, because in addition to the negative curvature space, we are introducing a flux $F_{y_{1} y_{2}}^{(3)}=q / y_{2}^{2}$ on these directions, and also adding a source for the operator dual to the $5 \mathrm{D}$ dilaton $\phi$. In the supersymmetric case analysed in [28], the effects of these deformations combine to preserve a twisted supersymmetry. The whole RG flow is supersymmetric, so on the field theory side the deformation of $\mathcal{N}=4 \mathrm{SYM}$ is preserving some supersymmetry. One would then not expect the field theory to have a scalar instability, and indeed the terms combine to leave us with flat directions for some of the field theory scalars [28]. Similarly, from the bulk perspective, the addition of the flux and deformation of the $\mathrm{S}^{5}$ (encoded in the $5 \mathrm{D}$ dilaton) will modify both the DBI and WZ components of a probe brane action, which could stabilise the brane.

We now present analyses from both points of view - using the Maldacena-Nunez approach to contruct the field theory, then confirming our results by a direct probe brane calculation.

\subsection{UV field theory analysis}

Let us analyze the field theory deformation for our general family of flows. The field theory includes six real scalars, transforming in the vector representation of the $\mathrm{SO}(6)$ R-symmetry group and the adjoint of $\mathrm{SU}(\mathrm{N})$. The consistent truncation we work with preserves an $\mathrm{SU}(2) \times \mathrm{U}(1)$ subgroup of $\mathrm{SO}(6)$, so it is convenient to organize the scalars into three complex scalar fields $\mathcal{W}_{1}, \mathcal{W}_{2}$ and $\mathcal{W}_{3}$, where $\mathcal{W}_{1}$ and $\mathcal{W}_{2}$ transform under the $\mathrm{SU}(2)$ and $\mathcal{W}_{3}$ transforms under the $\mathrm{U}(1)$. The bulk 5D dilaton $\phi$ corresponds to an operator $\mathcal{O}_{2}$ which is a symmetric traceless combination of the scalars transforming in the $\mathbf{2 0}$ of $\mathrm{SO}(6)$ [28],

$$
\mathcal{O}_{2}=\operatorname{Tr}\left\{\frac{2}{3}\left|\mathcal{W}_{3}\right|^{2}-\frac{1}{3}\left(\left|\mathcal{W}_{1}\right|^{2}+\left|\mathcal{W}_{2}\right|^{2}\right)\right\}
$$

The deformation we consider has a negative curvature in the $y_{1}, y_{2}$ directions and a flux of the $\tau^{3}$ component of the SU(2) gauge field through those directions, and a source for $\mathcal{O}_{2}$ with a coefficient $\bar{\lambda}$. This corresponds to a deformation of the scalar part of the field theory Lagrangian to

$$
S=\int d^{4} x\left\{\frac{1}{2}\left|D_{\mu} \mathcal{W}_{1}\right|^{2}+\frac{1}{2}\left|D_{\mu} \mathcal{W}_{2}\right|^{2}+\frac{1}{2}\left|\partial_{\mu} \mathcal{W}_{3}\right|^{2}-\frac{R}{12} \sum_{i}\left|\mathcal{W}_{i}\right|^{2}+\frac{3}{4} \bar{\lambda} R \mathcal{O}_{2}\right\}
$$


where $D_{\mu}=\partial_{\mu}+\mathrm{i} A_{\mu}$ is the gauge-covariant derivative with respect to the component of the $\mathrm{SU}(2)$ gauge field we turn on, and $R$ is the Ricci scalar of the two dimensional hyperbolic spacetime (note $R=-|R|<0$ ). Substituting in $A_{y_{1}}=q / y_{2}$, we have

$$
\begin{aligned}
S=\int d^{4} x & \left\{\frac{1}{2} \sum_{i}\left|\partial_{\mu} \mathcal{W}_{i}\right|^{2}-|R|\left(\frac{\bar{\lambda}}{2}-\frac{1}{12}\right)\left|\mathcal{W}_{3}\right|^{2}\right. \\
& \left.-|R|\left[\frac{Q^{2}}{8}-\left(\frac{\bar{\lambda}}{4}+\frac{1}{12}\right)\right]\left(\left|\mathcal{W}_{1}\right|^{2}+\left|\mathcal{W}_{2}\right|^{2}\right)\right\},
\end{aligned}
$$

where the normalization of the $Q^{2}$ term and the coefficient of $\bar{\lambda}$ have been fixed by reference to the supersymmetric case, which corresponds to $\bar{\lambda}=\frac{1}{6}$ and $Q=1$.

\subsection{Probe brane calculation}

We now want to explore this field theory from the bulk perspective. Holographically, Rsymmetry scalar fields correspond to inserting a brane with its four infinite dimensions parallel to an $r=$ const. section of the 5D space, and at a given position on the (possibly distorted) $S^{5}$. The effective action of such a probe brane is given by the sum of a geometric DBI term, and a topological WZ term:

$$
S=-T_{3} g_{s}^{-1} \int e^{-\Phi} \sqrt{-\operatorname{det}\left[\gamma_{A B}+\mathrm{F}_{A B}\right]} d^{4} \zeta+T_{3} \int C_{4}
$$

where $\zeta^{A}$ are the intrinsic coordinates on the brane worldvolume; $\gamma_{A B}$ the induced metric; $\mathrm{F}_{A B}=\mathrm{B}_{A B}+2 \pi \alpha^{\prime} F_{A B}$, the pullback of the 2-form field to the brane (zero in this background) and worldvolume gauge field (which we also set to zero); finally, $C_{4}$ is the pullback of the 4-form gauge potential onto the brane.

In order to compute this action, we first need the background geometry. The twisting introduced previously corresponds to a distortion of the $S^{5}$ in the reduction of the IIB SUGRA as described in [33]. ${ }^{2}$ Lifting the $5 \mathrm{D}$ solutions of $(2.5),(2.6)$ to $10 \mathrm{D}$, and writing

$$
\begin{array}{ll}
S=\sin \chi & \Delta=\xi^{2} S^{2}+\xi^{-1} C^{2} \\
C=\cos \chi & U=\xi S^{2}+\xi^{-2} C^{2}+\xi
\end{array}
$$

gives: ${ }^{3}$

$$
\begin{aligned}
d s^{2}= & \Delta^{\frac{1}{2}}\left(e^{2 F} d t^{2}-r^{2} d x^{2}-e^{2 d} \frac{d r^{2}}{r^{2}}-e^{2 h} \frac{d y_{1}^{2}+d y_{2}^{2}}{y_{2}^{2}}\right) \\
& -\xi^{-1} \Delta^{-\frac{1}{2}}\left[\Delta d \chi^{2}+\xi^{-1} S^{2}(d \eta-2 \mathcal{A})+\frac{1}{4} \xi^{2} C^{2} \sum_{i}\left(h^{(i)}\right)^{2}\right] \\
\mathbf{F}_{5}= & 2 U \epsilon_{5}+3 S C \xi^{-1} \star_{5} d \xi \wedge d \chi+\frac{C^{2}}{2 \sqrt{2}} \xi^{2} \star_{5} F_{2}^{(3)} \wedge \sigma^{(1)} \wedge \sigma^{(2)} \\
& -\frac{S C}{\sqrt{2}} \xi^{2} \star_{5} F_{2}^{(3)} \wedge h^{(3)} \wedge d \chi-2 S C \xi^{-4} \star_{5} \mathcal{F}_{2} \wedge d \chi \wedge(d \eta-2 \mathcal{A}),
\end{aligned}
$$

\footnotetext{
${ }^{2}$ Note that there are some factors of two between the variables used here and those of [33]: $(\phi)_{L P T}=\phi / 2$, $\left(g_{i}\right)_{L P T}=g_{i} / 2$, and $A_{L P T}=2 A$, where $A$ stands for either the $\mathrm{U}(1)$ or $\mathrm{SO}(3)$ gauge field.

${ }^{3}$ We have set $g_{1}=g_{2} / \sqrt{2}=2$ to match the conventions of [28], Gregory:2010gx
} 
the other form fields, the string dilaton and axion vanish. Here, $h^{(i)}$ are the left invariant forms on $S^{3}\left(\sigma^{(i)}\right)$ modified by the $\mathrm{SO}(3)$ gauge fields:

$$
h^{(i)}=\sigma^{(i)}-2 \sqrt{2} A^{(i)} .
$$

For constant $\xi$, we may reparametrize the squashed $S^{5}$ as

$$
\begin{aligned}
W_{1} & =\xi \cos \chi \cos \frac{\theta}{2} e^{\mathrm{i} \frac{\phi+\psi}{2}} \\
W_{2} & =\xi \cos \chi \sin \frac{\theta}{2} e^{\mathrm{i} \frac{\phi-\psi}{2}} \\
W_{3} & =\xi^{-1 / 2} \sin \chi e^{\mathrm{i} \eta}
\end{aligned}
$$

which, together with the obvious definitions of the gauge covariant differentiation for $W_{1,2}$ and $W_{3}$ give the metric of the additional dimensions as

$$
d s_{5}=-\xi^{-1} \Delta^{-\frac{1}{2}}\left[\left|D W_{1}\right|^{2}+\left|D W_{2}\right|^{2}+\left|\mathcal{D} W_{3}\right|^{2}\right]
$$

As $\xi$ changes from unity, we can see how the $S^{5}$ becomes distorted while maintaining an $\mathrm{SO}(3) \times \mathrm{U}(1)$ symmetry. Our $5 \mathrm{D}$ dilaton is thus a shape modulus for the $S^{5}$. Since $\xi \equiv 1$ for $\mathrm{AdS}_{5}$, it is now transparent how to deal with the degrees of freedom of the probe brane: we simply replace the ' $\xi$ ' in (4.9) with a radial variable $r(\zeta)$, and allow the remaining angular degrees of freedom of the brane to also depend on the brane coordinates $\zeta^{A}$. We will then expand the action for a slowly moving brane at large $r$ in the asymptotic $\mathrm{AdS}_{5}$ solution.

We start with the DBI part of the action

$$
S_{D B I} \propto-\int d^{4} \zeta \sqrt{-\operatorname{det} \gamma_{A B}}
$$

where

$$
\gamma_{A B}=\frac{\partial X^{a}}{\partial \zeta^{A}} \frac{\partial X^{b}}{\partial \zeta^{B}} g_{a b}
$$

with $X^{\mu}=\left[t, x, r(\zeta), y_{1}, y_{2}, \chi(\zeta), \eta(\zeta), \theta(\zeta), \phi(\zeta), \psi(\zeta)\right]$ being the brane's spacetime coordinates in terms of the intrinsic coordinates $\zeta$, for which we choose the gauge $\zeta^{A}=$ $\left(t, x, y_{1}, y_{2}\right)$. Thus

$$
\gamma_{A B}=\gamma_{A B}^{0}-\frac{1}{r^{2}}\left[D_{A} W_{1} \overline{D_{B} W_{1}}+D_{A} W_{2} \overline{D_{B} W_{2}}+\mathcal{D}_{A} W_{3} \overline{\mathcal{D}_{B} W_{3}}\right]
$$

where $\gamma_{A B}^{0}=\Delta^{\frac{1}{2}} \cdot \operatorname{diag}\left(e^{2 F},-r^{2},-\frac{e^{2 h}}{y_{2}^{2}},-\frac{e^{2 h}}{y_{2}^{2}}\right)$, the $1 / r^{2}$ factor arising because we have replaced $\xi$ with $r$ in (4.9). Hence,

$$
\sqrt{-\operatorname{det} \gamma_{A B}} \simeq \sqrt{-\operatorname{det} \gamma_{a b}^{0}}\left(1-\frac{1}{2 r^{2}} \gamma^{0 A B} D_{A} W_{i} \overline{D_{B} W_{i}}\right)
$$

(where we understand the covariant derivative in the sum to be the one relevant to the particular $W_{i}$ ). Since we are only interested in the leading order behaviour as we change $W_{i}$, we only require $\gamma^{0 A B}$ to leading order in $W_{i}$, i.e. at the $\mathrm{AdS}_{5}$ limit:

$$
\left.\gamma^{0 A B}\right|_{\text {AdS5 }}=\frac{1}{r^{2}} \cdot \operatorname{diag}\left(1,-1,-y_{2}^{2} e^{-2 h_{0}},-y_{2}^{2} e^{-2 h_{0}}\right)
$$


hence

$$
S_{D B I} \propto-\int d^{4} \zeta \frac{r \Delta}{y_{2}^{2}} e^{F+2 h}\left(1-\frac{1}{2 r^{4}} \sum_{i}\left|D_{\mu} W_{i}\right|^{2}\right)
$$

For the WZ term, note that although the 4 -form potential is rather involved for a general flow, we only require the leading order part parallel to the probe brane worldvolume, which can be found by integrating the $U$ function in (4.5). Putting this together, we see that

$$
S_{\text {eff }} \sim \int d^{4} \zeta\left\{-\Delta(\xi, \chi) \cdot r e^{F+2 h}\left(1-\frac{1}{2 r^{4}} \sum_{i}\left|D_{\mu} W_{i}\right|^{2}\right)+2 \int e^{F+d+2 h} \mathrm{U}(\xi, \chi) \mathrm{dr}\right\}
$$

We now expand this action in the asymptotic $\mathrm{AdS}_{5}$ region, but with one difference to the procedure followed in $\S$ 3.1.1: we need to consider a linear expansion in the case of finite volume of the 2D hyperbolic space, i.e. finite $h_{0}$. The full asymptotic solution together with corrected expansion up to $r^{-2}$ order reads

$$
\begin{aligned}
F & =\ln r, & d & =-\frac{e^{-2 h_{0}}}{6 r^{2}}, \\
h & =\ln r+h_{0}+\frac{e^{-2 h_{0}}}{4 r^{2}}, & \xi & =1+\frac{\sqrt{2}}{3} \frac{\lambda \ln r}{r^{2}}+\frac{\sqrt{2}}{3} \frac{\mu}{r^{2}} .
\end{aligned}
$$

Substituting these expressions into (4.17), and performing the integral for $U$, we see that all terms proportional to $\mu$ and $\lambda \ln r$ cancel leaving

$$
S_{\text {eff }} \sim \int d^{4} \zeta\left\{\frac{1}{2} e^{2 h_{0}} \sum_{i}\left|D_{\mu} W_{i}\right|^{2}-\frac{\lambda}{3 \sqrt{2}} e^{2 h_{0}}\left(2 S^{2}-C^{2}\right) r^{2}+\frac{1}{6} r^{2}\right\}
$$

It is easy to see that we can identify

$$
\left(2 S^{2}-C^{2}\right) r^{2}=3 \mathcal{O}_{2}, \quad r^{2}=\sum_{i}\left|W_{i}\right|^{2}
$$

and noting the relation between our $\lambda$ and $\bar{\lambda},(3.14)$, as well as the curvature of the $2 \mathrm{D}$ hyperbolic space, $R=-2 e^{-2 h_{0}}$, we get

$$
S_{\text {eff }} \propto \int d^{4} \zeta e^{2 h_{0}}\left\{\frac{1}{2} \sum_{i}\left|D_{\mu} W_{i}\right|^{2}-\frac{3}{4} \bar{\lambda} R \mathcal{O}_{2}+\frac{1}{12} R \sum_{i}\left|W_{i}\right|^{2}\right\}
$$

which coincides with the expression for the field theory effective action (4.2) precisely. ${ }^{4}$

\subsection{Stability and Lifshitz dual field theories}

Having obtained the field theory action, (4.3), we now analyse the scalar stability. In order to have stable potential for the $\mathcal{W}_{3}$ field, we should have

$$
\frac{1}{2} \bar{\lambda}-\frac{1}{12} \geq 0 \Rightarrow \bar{\lambda} \geq \frac{1}{6}
$$

\footnotetext{
${ }^{4}$ Indeed, the uplift of the AdS flows can be generalised in the context of solutions in $D=10,11$ dual to $\mathcal{N}=2$ SCFT's, as studied in [39, 40]. (We thank Jerome Gauntlett for pointing this out.)
} 
While for the twisted fields $\mathcal{W}_{1}$ and $\mathcal{W}_{2}$ we should have

$$
\frac{Q^{2}}{8}-\left(\frac{1}{4} \bar{\lambda}+\frac{1}{12}\right) \geq 0
$$

For the supersymmetric case, both these bounds are automatically saturated (by our choice of normalization in matching operator sources to bulk modes), reproducing the flat directions of [28].

For $\mathrm{AdS}_{3}$ solutions we know that in the $\mathrm{AdS}_{3}$ region $Q^{2}=\varphi \sqrt{2}-1$, and, by numerical analysis we determine $\bar{\lambda}$ as a function of the value of $\varphi$ in the $\mathrm{AdS}_{3}$ region. The stability criterion for the $\mathcal{W}_{3}$ field, $\bar{\lambda} \geq 1 / 6$, which corresponds to $\varphi \geq \sqrt{2}$. Meanwhile, (4.23) provides an upper bound on $\varphi$, as $\bar{\lambda}$ increases more rapidly than $Q^{2}$ along the family of $\mathrm{AdS}_{3}$ flows. Numerically, we find that the $\mathrm{AdS}_{3}$ solutions with

$$
\varphi \in[\sqrt{2}, \sim 3.26]
$$

result from an RG flow from a field theory in the UV where the field theory deformation is not introducing a field theory scalar instability. The corresponding region for the charge $Q$ is

$$
Q^{2} \in[1, \sim 3.61]
$$

Disappointingly, for the Lifshitz solutions we found numerically that none of the solutions involve flows with $\bar{\lambda} \geq 1 / 6$. The flows on the untilded branch do approach $\bar{\lambda} \rightarrow 1 / 6$ when $z \rightarrow 2$, but $Q \rightarrow 0$ in this limit, so even if we are nearly satisfying the stability condition for $\mathcal{W}_{3}$ in the limit, the condition for $\mathcal{W}_{1}$ and $\mathcal{W}_{2}$ is badly violated. Thus, none of our Lifshitz solutions is obtained as an RG flow from a stable UV field theory, and we cannot use these RG flows to define the field theory dual to the IR fixed points.

This UV instability does not necessarily imply that the IR fixed points are ill-defined, just that this approach to constructing them has failed. There are solutions on the $\mathrm{Li}_{3}$ branch for which we did not have evidence of a supergravity instability which are still candidates for having a dual field theory; but we will have to look elsewhere for a top-down definition of this field theory.

\section{Acknowledgments}

We are grateful for useful conversations with Mukund Rangamani, and for collaboration with Ludovic Plante on an early version of this work. PB would like to thank Perimeter Institute for hospitality. PB is supported by an EPSRC International Doctoral Scholarship, RG and SFR are supported in part by STFC (Consolidated Grant ST/J000426/1). RG is also supported by the Wolfson Foundation and Royal Society, and Perimeter Institute for Theoretical Physics. Research at Perimeter Institute is supported by the Government of Canada through Industry Canada and by the Province of Ontario through the Ministry of Research and Innovation. 


\section{A Additional AdS solutions}

In the main text we assumed that the topologically charged part of the fluxes, i.e. the flux through the compact hyperbolic space, only involved the $\mathrm{SU}(2)$ gauge field, as this is the only possibility for the Lifshitz solutions [11]. However, more generally the abelian field could also have a topological flux. Here we will briefly discuss constructing more general $\mathrm{AdS}_{3}$ geometries using this freedom. These solutions were also obtained in a more systematic analysis in $[37,38]$.

Introducing the following more general ansatz for the gauge fields

$$
\begin{aligned}
\mathcal{F}_{y_{1} y_{2}} & =\frac{q_{1}}{y_{2}^{2}}, \\
F_{y_{1} y_{2}}^{(3)} & =\frac{q_{2}}{y_{2}^{2}},
\end{aligned}
$$

together with the standard ansatz for the metric (2.5) with $r$-independent constants $d_{0}$ and $h_{0}$ and $F(\rho)=\rho$, gives rise to the following system of equations

$$
\begin{aligned}
2 e^{-2 D_{0}} & =\frac{1}{6}\left(\varphi_{0}^{-\frac{2}{3}}+2 \sqrt{2} \varphi_{0}^{\frac{1}{3}}\right)+\frac{2}{3} \varphi^{\frac{2}{3}} Q_{2}^{2} e^{-4 H_{0}}+\frac{2}{3} \varphi^{-\frac{4}{3}} Q_{1}^{2} e^{-4 H_{0}}, \\
e^{-2 H_{0}} & =\frac{1}{6}\left(\varphi_{0}^{-\frac{2}{3}}+2 \sqrt{2} \varphi_{0}^{\frac{1}{3}}\right)-\frac{4}{3} \varphi_{0}^{\frac{2}{3}} Q_{2}^{2} e^{-4 H_{0}}-\frac{4}{3} \varphi_{0}^{-\frac{4}{3}} Q_{1}^{2} e^{-4 H_{0}}, \\
0 & =\frac{1}{2}\left(-\varphi_{0}^{-\frac{2}{3}}+\sqrt{2} \varphi_{0}^{\frac{1}{3}}\right)-2 \varphi_{0}^{\frac{2}{3}} Q_{2}^{2} e^{-4 H_{0}}+4 \varphi_{0}^{-\frac{4}{3}} Q_{1}^{2} e^{-4 H_{0}},
\end{aligned}
$$

where $Q_{1}=q_{1} g_{1}$. Solving this system gives us a two-parameter family of $\mathrm{AdS}_{3}$ solutions,

$$
\begin{aligned}
e^{-2 D_{0}} & =f_{D}\left(Q_{1}, Q_{2}\right), \\
e^{-2 H_{0}} & =f_{H}\left(Q_{1}, Q_{2}\right), \\
\varphi_{0} & =f_{\varphi}\left(Q_{1}, Q_{2}\right),
\end{aligned}
$$

which will coincide with $(2.15)$ if we put $Q_{1}=0, g_{1}=2, g_{2}=2 \sqrt{2}$ and $Q_{2}=Q$. These solutions are supersymmetric if

$$
Q_{1}+Q_{2}=1
$$

Field theory duals for two points in this family $\left(Q_{1}=1\right.$ and $\left.Q_{2}=1\right)$ were discussed through twisting in [28]. There it was also pointed out that the field theory description of the general supersymmetric solution of (A.2) would involve some fields acquiring fractional spins during twisting.

Open Access. This article is distributed under the terms of the Creative Commons Attribution License (CC-BY 4.0), which permits any use, distribution and reproduction in any medium, provided the original author(s) and source are credited.

\section{References}

[1] J. M. Maldacena, The Large $N$ limit of superconformal field theories and supergravity, Int. J. Theor. Phys. 38 (1999) 1113 [hep-th/9711200]. 
[2] S. Sachdev, Condensed Matter and AdS/CFT, Lect. Notes Phys. 828 (2011) 273 [arXiv:1002.2947 [hep-th]].

[3] J. McGreevy, Holographic duality with a view toward many-body physics, Adv. High Energy Phys. 2010 (2010) 723105 [arXiv:0909.0518 [hep-th]].

[4] S. A. Hartnoll, Lectures on holographic methods for condensed matter physics, Class. Quant. Grav. 26 (2009) 224002 [arXiv:0903.3246 [hep-th]].

[5] S. Kachru, X. Liu and M. Mulligan, Gravity duals of Lifshitz-like fixed points, Phys. Rev. D 78 (2008) 106005 [arXiv:0808.1725 [hep-th]].

[6] M. Taylor, Non-relativistic holography, arXiv:0812.0530 [hep-th].

[7] K. Balasubramanian and K. Narayan, Lifshitz spacetimes from AdS null and cosmological solutions, JHEP 1008 (2010) 014 [arXiv:1005.3291 [hep-th]].

[8] A. Donos and J. P. Gauntlett, Lifshitz Solutions of $D=10$ and $D=11$ supergravity, JHEP 1012 (2010) 002 [arXiv:1008.2062 [hep-th]].

[9] D. Cassani and A. F. Faedo, Constructing Lifshitz solutions from AdS, JHEP 1105 (2011) 013 [arXiv:1102.5344 [hep-th]].

[10] P. Dey and S. Roy, From AdS to Schrödinger/Lifshitz dual space-times without or with hyperscaling violation, JHEP 1311 (2013) 113 [arXiv:1306.1071 [hep-th]].

[11] R. Gregory, S. L. Parameswaran, G. Tasinato and I. Zavala, Lifshitz solutions in supergravity and string theory, JHEP 1012 (2010) 047 [arXiv:1009.3445 [hep-th]].

[12] H. Singh, Special limits and non-relativistic solutions, JHEP 1012 (2010) 061 [arXiv:1009.0651 [hep-th]].

[13] H. Singh, Lifshitz/Schródinger Dp-branes and dynamical exponents, JHEP 1207 (2012) 082 [arXiv:1202.6533 [hep-th]].

[14] A. Donos, J. P. Gauntlett, N. Kim and O. Varela, Wrapped M5-branes, consistent truncations and AdS/CMT, JHEP 1012 (2010) 003 [arXiv:1009.3805 [hep-th]].

[15] H. Braviner, R. Gregory and S. F. Ross, Flows involving Lifshitz solutions, Class. Quant. Grav. 28 (2011) 225028 [arXiv:1108.3067 [hep-th]].

[16] H. Singh, Holographic flows to IR Lifshitz spacetimes, JHEP 1104 (2011) 118 [arXiv:1011.6221 [hep-th]].

[17] G. Bertoldi, B. A. Burrington and A. W. Peet, Thermal behavior of charged dilatonic black branes in AdS and UV completions of Lifshitz-like geometries, Phys. Rev. D 82 (2010) 106013 [arXiv:1007.1464 [hep-th]].

[18] S. S. Gubser and A. Nellore, Ground states of holographic superconductors, Phys. Rev. D 80 (2009) 105007 [arXiv:0908.1972 [hep-th]].

[19] K. Goldstein, N. Iizuka, S. Kachru, S. Prakash, S. P. Trivedi and A. Westphal, Holography of Dyonic Dilaton Black Branes, JHEP 1010 (2010) 027 [arXiv:1007.2490 [hep-th]].

[20] S. A. Hartnoll and A. Tavanfar, Electron stars for holographic metallic criticality, Phys. Rev. D 83 (2011) 046003 [arXiv:1008.2828 [hep-th]].

[21] J. T. Liu and Z. Zhao, Holographic Lifshitz flows and the null energy condition, [arXiv:1206.1047 [hep-th]]. 
[22] H. Singh, Lifshitz to AdS flow with interpolating p-brane solutions, JHEP 1308 (2013) 097 [arXiv:1305.3784 [hep-th]].

[23] S. Kachru, N. Kundu, A. Saha, R. Samanta and S. P. Trivedi, Interpolating from Bianchi Attractors to Lifshitz and AdS Spacetimes, JHEP 1403 (2014) 074 [arXiv:1310.5740 [hep-th] ].

[24] P. Dey and S. Roy, Interpolating solution from $A d S_{5}$ to hyperscaling violating Lifshitz space-time, [arXiv:1406.5992 [hep-th]].

[25] K. Uzawa and K. Yoshida, Dynamical Lifshitz-type solutions and aging phenomena, Phys. Rev. D 87 (2013) 106003 [arXiv:1302.5224 [hep-th]].

[26] Y. Korovin, K. Skenderis and M. Taylor, Lifshitz from AdS at finite temperature and top down models, JHEP 1311 (2013) 127 [arXiv:1306.3344 [hep-th]].

[27] Y. Korovin, K. Skenderis and M. Taylor, Lifshitz as a deformation of Anti-de Sitter, JHEP 1308 (2013) 026 [arXiv:1304.7776 [hep-th]].

[28] J. M. Maldacena and C. Nunez, Supergravity description of field theories on curved manifolds and a no go theorem, Int. J. Mod. Phys. A 16 (2001) 822 [hep-th/0007018].

[29] N. Seiberg and E. Witten, The D1 / D5 system and singular CFT, JHEP 9904 (1999) 017 [hep-th/9903224].

[30] E. Witten and S. -T. Yau, Connectedness of the boundary in the AdS / CFT correspondence, Adv. Theor. Math. Phys. 3 (1999) 1635 [hep-th/9910245].

[31] P. Breitenlohner and D. Z. Freedman, Stability in Gauged Extended Supergravity, Annals Phys. 144 (1982) 249.

[32] L. J. Romans, Gauged $N=4$ Supergravities in Five-dimensions and Their Magnetovac Backgrounds, Nucl. Phys. B 267 (1986) 433.

[33] H. Lu, C. N. Pope and T. A. Tran, Five-dimensional N=4, SU(2) x U(1) gauged supergravity from type IIB, Phys. Lett. B 475 (2000) 261 [hep-th/9909203].

[34] L. Girardello, M. Petrini, M. Porrati and A. Zaffaroni, Novel local CFT and exact results on perturbations of $N=4$ superYang Mills from AdS dynamics, JHEP 9812 (1998) 022 [hep-th/9810126].

[35] D. Z. Freedman, S. S. Gubser, K. Pilch and N. P. Warner, Renormalization group flows from holography supersymmetry and a c theorem, Adv. Theor. Math. Phys. 3 (1999) 363 [hep-th/9904017].

[36] A. J. Amsel, G. T. Horowitz, D. Marolf and M. M. Roberts, No Dynamics in the Extremal Kerr Throat, JHEP 0909 (2009) 044 [arXiv:0906.2376 [hep-th]].

[37] F. Benini and N. Bobev, Exact two-dimensional superconformal R-symmetry and c-extremization, Phys. Rev. Lett. 110 (2013) 6, 061601 [arXiv:1211.4030 [hep-th]].

[38] F. Benini and N. Bobev, Two-dimensional SCFTs from wrapped branes and c-extremization, JHEP 1306 (2013) 005 [arXiv:1302.4451 [hep-th]].

[39] J. P. Gauntlett and O. Varela, Consistent Kaluza-Klein reductions for general supersymmetric AdS solutions, Phys. Rev. D 76 (2007) 126007 [arXiv:0707.2315 [hep-th]].

[40] J. P. Gauntlett and O. Varela, $D=5 \mathrm{SU}(2) \times \mathrm{U}(1)$ Gauged Supergravity from $\mathrm{D}=11$ Supergravity, JHEP 0802 (2008) 083 [arXiv:0712.3560 [hep-th]]. 